\title{
COMPLETELY BOUNDED HOMOMORPHISMS OF OPERATOR ALGEBRAS
}

\author{
VERN I. PAULSEN ${ }^{1}$
}

\begin{abstract}
Let $A$ be a unital operator algebra. We prove that if $\rho$ is a completely bounded, unital homomorphism of $A$ into the algebra of bounded operators on a Hilbert space, then there exists a similarity $S$, with $\left\|S^{-1}\right\|$. $\|S\|=\|\rho\|_{\mathrm{cb}}$, such that $S^{-1} \rho(\cdot) S$ is a completely contractive homomorphism. We also show how Rota's theorem on operators similar to contractions and the result of Sz.-Nagy and Foias on the similarity of $\rho$-dilations to contractions can be deduced from this result.
\end{abstract}

1. Introduction. In [6] we proved that a homomorphism $\rho$ of an operator algebra is similar to a completely contractive homomorphism if and only if $\rho$ is completely bounded. It was known that if $S$ is such a similarity, then $\|S\| \cdot\left\|S^{-1}\right\| \geq$ $\|\rho\|_{\mathrm{cb}}$. However, at the time we were unable to determine if one could choose the similarity such that $\|S\| \cdot\left\|S^{-1}\right\|=\|\rho\|_{\mathrm{cb}}$. When the operator algebra is a $C^{*}$ algebra then Haagerup had shown [3] that such a similarity could be chosen. The purpose of the present note is to prove that for a general operator algebra, there exists a similarity $S$ such that $\|S\| \cdot\left\|S^{-1}\right\|=\|\rho\|_{\mathrm{cb}}$.

Completely contractive homomorphisms are central to the study of the representation theory of operator algebras, since they are precisely the homomorphisms that can be dilated to a *-representation on some larger Hilbert space of any $C^{*}$ algebra which contains the operator algebra. For $C^{*}$-algebras the sets of contractive homomorphisms, completely contractive homomorphisms, and $*$-homomorphisms coincide.

The main result of this paper also gives, at least, a theoretical answer to certain minimization problems. Suppose, for example, that $T$ is an operator on a Hilbert space that is similar to a contraction; then $\inf \left\{\|S\| \cdot\left\|S^{-1}\right\|:\left\|S^{-1} T S\right\| \leq 1\right\}$ is attained and equal to $\|\rho\|_{\mathrm{cb}}$, where $\rho$ is the homomorphism of the disk algebra defined by $\rho(f)=f(T)$. An extensive study of this infimum was undertaken in [5], and it was studied in [2] for certain Toeplitz operators.

Finally, we end this note by showing how Rota's theorem [7] that every operator with spectral radius less than 1 is similar to a contraction, and the result of Sz.Nagy and Foias [8] that every operator with a $\rho$-dilation is similar to a contraction, can be easily deduced from our result. These new proofs give a unified principle of estimating the above infimum for both of these classes of operators.

2. The similarity theorem. Let $H$ denote a Hilbert space, $L(H)$ the bounded linear operators on $H, B$ a unital $C^{*}$-algebra, and let $A$ be a subalgebra of $B$ containing the unit of $B$. We call $A$ an operator algebra. We let $M_{n}$ denote the

Received by the editors October 18, 1983.

1980 Mathematics Subject Classification. Primary 46L05.

${ }^{1}$ Research supported in part by a grant from the NSF. $0002-9939 / 84 \$ 1.00+\$ .25$ per page 
$n \times n$ complex matrices, and $M_{n}(A)$ the tensor product of $A$ and $M_{n}$. We endow $M_{n}(A)$ with the norm that it inherits as a subspace of the $C^{*}$-algebra $M_{n}(B)$.

Given a map $\rho: A \rightarrow L(H)$, we define maps $\rho_{n}: M_{n}(A) \rightarrow L(H+\cdots+H)(n$ copies) by $\rho_{n}\left(\left(a_{i j}\right)\right)=\left(\rho\left(a_{i j}\right)\right)$ for $\left(a_{i j}\right)$ in $M_{n}(A)$. We call $\rho$ completely bounded provided that $\sup _{n}\left\|\rho_{n}\right\|$ is finite and we let $\|\rho\|_{\mathrm{cb}}$ denote this supremum. If $\|\rho\|_{\mathrm{cb}} \leq$ 1 , then we say that $\rho$ is completely contractive. By $[\mathbf{1}]$, a homomorphism $\rho$ of an operator algebra $A$ into $L(H)$ is completely contractive if and only if it can be dilated to $B$, that is, if and only if there exists a *-representation $\Pi: B \rightarrow L(K)$ for some Hilbert space $K$, containing $H$, such that $\rho(a)=\left.P \Pi(a)\right|_{H}$ for all $a$ in $A$, where $P$ denotes the projection of $K$ onto $H$.

THEOREM. Let $A$ be an operator algebra contained in a $C^{*}$-algebra $B$, and let $\rho: A \rightarrow L(H)$ be a unital, completely bounded homomorphism. Then there exists an invertible operator $S$, with $\|S\| \cdot\left\|S^{-1}\right\|=\|\rho\|_{\mathrm{cb}}$ such that $S^{-1} \rho(\cdot) S$ is a completely contractive homomorphism.

ProOF. By the generalization of Stinespring's Theorem [6, Theorem 2.8] and by [6, Theorem 2.4], there exists a Hilbert space $K$, a $*$-homomorphism $\Pi: B \rightarrow L(K)$, and two bounded operators $V_{i}: H \rightarrow K, i=1,2$, with $\left\|V_{1}\right\| \cdot\left\|V_{2}\right\|=\|\rho\|_{\mathrm{cb}}$ such that $\rho(a)=V_{1}^{*} \Pi(a) V_{2}$, for $a$ in $A$.

Following [4, p. 1030], for $h \in H$, we define

$$
|h|=\inf \left\{\left\|\sum \Pi\left(a_{i}\right) V_{2} h_{i}\right\|: \sum \rho\left(a_{i}\right) h_{i}=h, a_{i} \in A, h_{i} \in H\right\},
$$

where the infimum is taken over finite sums. By a minor modification of the arguments in $[\mathbf{4}$, p. 1030], one obtains that $|\cdot|$ is a norm on $H$ and $(H,|\cdot|)$ is a Hilbert space.

If $h=\sum \rho\left(a_{i}\right) h_{i}$, then

$$
\begin{aligned}
\|h\| & =\left\|\sum \rho\left(a_{i}\right) h_{i}\right\|=\left\|\sum V_{1}^{*} \Pi\left(a_{i}\right) V_{2} h_{i}\right\| \\
& \leq\left\|V_{1}^{*}\right\| \cdot\left\|\sum \Pi\left(a_{i}\right) V_{2} h_{i}\right\| .
\end{aligned}
$$

Thus, $\|h\| \leq\left\|V_{1}^{*}\right\| \cdot|h|$. Similarly, $\rho(1) h=h$ yields $|h| \leq\left\|V_{2}\right\| \cdot\|h\|$. Thus, if we define $S:(H,|\cdot|) \rightarrow(H,\|\cdot\|)$ to be the identity, then $S$ is invertible and

$$
\left\|S^{-1}\right\| \cdot\|S\| \leq\left\|V_{1}^{*}\right\| \cdot\left\|V_{2}\right\|=\|\rho\|_{\mathrm{cb}} .
$$

To complete the proof of the theorem it will be sufficient to prove that $S^{-1} \rho(\cdot) S$ is completely contractive, since then $\left\|S^{-1}\right\| \cdot\|S\| \geq\|\rho\|_{\mathrm{cb}}$ necessarily.

Let $a \in A$, and let $h=\sum \rho\left(a_{i}\right) h_{i}$. Then

$$
|\rho(a) h| \leq\left\|\sum \Pi\left(a a_{i}\right) V_{2} h_{i}\right\| \leq\|a\| \cdot\left\|\sum \Pi\left(a_{i}\right) V_{2} h_{i}\right\|,
$$

so $|\rho(a) h| \leq\|a\| \cdot|h|$. Thus, we obtain that $S^{-1} \rho(\cdot) S$ is contractive.

To see that $S^{-1} \rho(\cdot) S$ is completely contractive, fix an integer $n$, let $\hat{H}=H+\cdots+$ $H$ ( $n$ copies) and let $|\cdot|_{n}$ denote the norm on $\hat{H}$ given by $|\hat{h}|_{n}=\left|h_{1}\right|^{2}+\cdots+\left|h_{n}\right|^{2}$, $\hat{h}=\left(h_{1}, \ldots, h_{n}\right)$. We must prove that if $\hat{a}=\left(a_{i, j}\right) \in M_{n}(A)$ then $\left|\rho_{n}(\hat{a}) \hat{h}\right|_{n} \leq$ $\|\hat{a}\| \cdot|\hat{h}|_{n}$ for $\hat{h} \in \hat{H}$.

To this end, consider $\rho_{n}: M_{n}(A) \rightarrow L(\hat{H})$ where $\hat{H}$ is endowed with its old norm, i.e., $\|\hat{h}\|^{2}=\left\|h_{1}\right\|^{2}+\cdots+\left\|h_{n}\right\|^{2}$. Since $\rho$ is completely bounded, $\rho_{n}$ will 
be completely bounded and, in fact, $\left\|\rho_{n}\right\|_{\mathrm{cb}}=\|\rho\|_{\mathrm{cb}}$. Thus, by the first part of our argument we may endow $\hat{H}$ with yet another norm $|\cdot|_{(n)}$ such that $\rho_{n}(\cdot)$ is contractive in this norm, i.e., $\left|\rho_{n}(\hat{a}) \hat{h}\right|_{(n)} \leq\|\hat{a}\| \cdot|\hat{h}|_{(n)}$.

To construct $|\cdot|_{(n)}$, all we need is a Stinespring representation of $\rho_{n}$. For such a representation, consider $\Pi_{n}: M_{n}(B) \rightarrow L(\hat{K}), \hat{K}=K+\cdots+K(n$ copies $)$ and $\hat{V}_{i}: \hat{H} \rightarrow \hat{K}$ defined by $\hat{V}_{i}\left(h_{1}, \ldots, h_{n}\right)=\left(V_{i} h_{1}, \ldots, V_{i} h_{n}\right), i=1,2$. It is easily seen that $\rho_{n}(\hat{a})=\hat{V}_{1} \Pi_{n}(\hat{a}) \hat{V}_{2}$ for $\hat{a} \in M_{n}(A)$. Thus, we may set

$$
|\hat{h}|_{(n)}=\inf \left\{\left\|\sum \Pi_{n}\left(\hat{a}_{i}\right) \hat{V}_{2} \hat{h}_{i}\right\|: \sum \rho_{n}\left(\hat{a}_{i}\right) \hat{h}_{i}=\hat{h}\right\},
$$

and $\rho_{n}$ will be contractive in this norm.

We claim that with these choices $|\hat{h}|_{(n)}=|\hat{h}|_{n}$, which will complete the proof of the theorem.

To prove the claim fix $\varepsilon>0$, let $\hat{a}_{k}=\left(a_{i, j, k}\right) \in M_{n}(A), \hat{h}_{k}=\left(h_{1, k}, \ldots, h_{n, k}\right) \in$ $\hat{H}$ be such that, $\sum \rho_{n}\left(\hat{a}_{k}\right) \hat{h}_{k}=\hat{h}$, and $|\hat{h}|_{(n)}^{2}+\varepsilon \geq\left\|\sum \Pi_{n}\left(\hat{a}_{k}\right) \hat{V}_{2} \hat{h}_{k}\right\|^{2}$. We then have that

$$
|\hat{h}|_{(n)}^{2}+\varepsilon \geq \sum_{i=1}^{n}\left\|\sum_{k} \sum_{j=1}^{n} \Pi\left(a_{i, j, k}\right) V_{2} h_{j, k}\right\|^{2} \geq \sum_{i=1}^{n}\left|h_{i}\right|^{2}=|\hat{h}|_{n}^{2},
$$

and so $|\hat{h}|_{(n)} \geq|\hat{h}|_{n}$. The other inequality follows similarly. This completes the proof of the theorem.

To see how Rota's Theorem [7] follows from the above, let $T$ be an operator whose spectrum is contained in the open unit disk. Recall that by the Riesz functional calculus, if $f(z)$ is a polynomial, then

$$
f(T)=\frac{1}{2 \Pi i} \int_{\Gamma} f(z)(T-z I)^{-1} d z
$$

where $\Gamma=\{z:|z|=1\}$. Setting $\rho(f)=f(T)$, and letting $\|f\|=\sup \{|f(z)|:|z|=$ $1\}$, we have that $\|\rho(f)\| \leq K\|f\|$, where

$$
K=\frac{1}{2 \Pi} \int_{\Gamma}\left\|(T-z I)^{-1}\right\| d|z|
$$

Thus, $\rho$ extends to a bounded homomorphism of the disk algebra. To see that $\rho$ is completely bounded (here we are thinking of the disk algebra as a subalgebra of the $C^{*}$-algebra of continuous functions on the circle), observe that for an $n \times n$ matrix of polynomials,

$$
\begin{aligned}
\left(f_{i, j}(T)\right) & =\frac{1}{2 \Pi i} \int\left(f_{i, j}(z)\left(T-z I^{-1}\right)\right) d z \\
& =\frac{1}{2 \Pi i} \int\left(f_{i, j}(z)\right)(\hat{T}-z \hat{I}) d z,
\end{aligned}
$$

where $\hat{T}$ is the direct sum of $n$ copies of $T$. Since $\left\|(T-z I)^{-1}\right\|=\left\|(\hat{T}-z \hat{I})^{-1}\right\|$, we have

$$
\left\|\left(f_{i, j}(T)\right)\right\| \leq K\left\|\left(f_{i, j}(z)\right)\right\|
$$

and so $\rho$ is completely bounded with $\|\rho\|_{\text {cb }} \leq K$. Hence, there is an invertible operator $S$ such that $\left\|S^{-1}\right\| \cdot\|S\| \leq K$ and $\left\|S^{-1} T S\right\|=\left\|S^{-1} \rho(z) S\right\| \leq\|z\|=1$. 
As a second application we mention the $\rho$-dilations considered in Sz.-Nagy and Foias [8]. An operator $T$ in $L(H)$ has a $\rho$-dilation if there is a unitary $U$ acting on $K, H$ contained in $K$, such that $T^{n}=\left.\rho P U^{n}\right|_{H}, n \geq 1$, where $P$ is the projection of $K$ onto $H$. For $f$ in the disk algebra, define $\phi(f)=\left.P f(U)\right|_{H}$, and $\Psi(f)=f(0) \cdot I$. One easily sees that $\phi$ and $\Psi$ are complete contractions.

Finally, setting $\gamma(f)=f(T)=\rho \phi(f)+(1-\rho) \Psi(f)$, we have that $\gamma$ is a completely bounded homomorphism, and $\|\gamma\|_{\mathrm{cb}} \leq 2 \rho-1$. Thus, there is an invertible $S$, $\left\|S^{-1}\right\| \cdot\|S\| \leq 2 \rho-1$, such that $S^{-1} T S$ is a contraction.

\section{REFERENCES}

1. W. B. Arveson, Subalgebras of $C^{*}$-algebras, Acta Math. 123 (1969), 141-224.

2. D. N. Clark, Toeplitz operators and k-spectral sets, Indiana Univ. Math. J. 33 (1984), 127-141.

3. U. Haagerup, Solution of the similarity problem for cyclic representations of $C^{*}$-algebras, Ann. of Math. 118 (1983), 215-240.

4. J. A. R. Holbrook, Spectral dilations and polynomially bounded operators, Indiana Univ. Math. J. 20 (1971), 1027-1034.

5. __ Distortion coefficients for crypto-contractions, Linear Algebra Appl. 18 (1977), 229-256.

6. V. I. Paulsen, Every completely polymomially bounded operator is similar to a contraction, J. Funct. Anal. 55 (1984), 1-17.

7. G.-C. Rota, On models for linear operators, Comm. Pure Appl. Math. 13 (1960), 468-472.

8. B. Sz.-Nagy and C. Foias, Harmonic analysis of operators on Hilbert space, American Elsevier, New York, 1970.

Department of Mathematics, University of Houston, Houston, TeXas 77004 